\title{
Emotion-inducing approaching sounds shape the boundaries of multisensory peripersonal space
}

Francesca Ferri ${ }^{1}$, Ana Tajadura-Jiménez ${ }^{2}$, Aleksander Väljamäe ${ }^{3}$, Roberta Vastano ${ }^{4,5}$ \& Marcello Costantini ${ }^{1,5,6}$

${ }^{1}$ Mind, Brain Imaging and Neuroethics, University of Ottawa, Institute of Mental Health Research, Ottawa, ON, Canada

${ }^{2}$ UCL Interaction Centre (UCLIC), University College London, University of London, London WC1E 6BT, UK

3 Department of Behavioural Sciences and Learning, Linköping University, Linköping, Sweden.

${ }^{4}$ Italian Institute of Technology (IIT) Department of Robotics, Brain and Cognitive Sciences

5 Department of Neuroscience, Imaging and Clinical Science, University G. d'Annunzio, Chieti, Italy

${ }^{6}$ Institute for Advanced Biomedical Technologies - ITAB, University G. d'Annunzio, Chieti, Italy

Short title: Emotion and peripersonal space

\section{Corresponding author:}

Francesca Ferri: Mind, Brain Imaging and Neuroethics, University of Ottawa, Institute of Mental Health Research, Ottawa, ON, Canada. Email: Francesca.Ferri@theroyal.ca 

Ferri et al.

\begin{abstract}
In order to survive in a complex environment, inhabited by potentially threatening and noxious objects or living beings, we need to constantly monitor our surrounding space, especially in the vicinity of our body. Such a space has been commonly referred to as one's 'peripersonal space' (PPS). In this study we investigated whether emotion-inducing approaching sound sources impact the boundaries of PPS. Previous studies have indeed showed that the boundaries of PPS are not fixed but modulate according to properties of stimuli in the surrounding environment. In Experiment 1, participants performed a simple tactile detection task of targets presented to their right hand. Concurrently, they were presented with intensity-changing task-irrelevant artificial sound sources perceived as approaching toward their body. The physical properties of the sound elicited emotional responses of either neutral or negative valence. Results showed larger PPS when the approaching stimulus had negative as compared to neutral emotional valence. In Experiment 2, we used ecological sounds which content (i.e., psychological associations to the sound producing source), rather than physical properties, elicited emotional responses of negative, positive or neutral valence. In agreement with results from experiment 1, we found larger PPS when the approaching stimuli had negative emotional valence as compared to both neutral and positive ones. Results are discussed within the theoretical framework that conceives PPS as a safety zone around one's body.
\end{abstract}

Keywords: peripersonal space, multisensory integration, emotion, auditory sources, approaching, looming sounds 

Ferri et al.

\section{Introduction}

The term Peripersonal space (PPS), as used in cognitive neuroscience research, commonly refers to as a multisensory space around our body (Rizzolatti, Fadiga, Fogassi, \& Gallese, 1997). In the field of social psychology the term "Personal space" is often used to define the emotionally-tinged zone around the human body that people experience as "their space" (Sommer, 1959) and which others cannot intrude without arousing discomfort (Hayduk, 1983). Evidence of the multisensory coding of PPS was firstly provided by electrophysiological single cell recording in the monkey brain (Rizzolatti, Scandolara, Matelli, \& Gentilucci, 1981). In 1981 Rizzolatti and colleagues described visuo-tactile neurons in the periarcuate cortex that selectively responded to stimuli presented in the space immediately around the animal (Rizzolatti, et al., 1981). Later studies of PPS coding identified neurons integrating somatosensory information with either visual or acoustical information within PPS in the ventral premotor cortex (Rizzolatti, et al., 1981), including the polysensory zone PZ (Graziano \& Gandhi, 2000), in the ventral intraparietal sulcus (Avillac, Deneve, Olivier, Pouget, \& Duhamel, 2005; Duhamel, Bremmer, Ben Hamed, \& Graf, 1997), in the parietal areas 7b, and in the putamen (Graziano \& Gross, 1993). The existence of a similar fronto-parietal system for the multisensory coding of PPS in the human brain has been shown by different neuroimaging and neurophysiological studies (Bremmer, et al., 2001; Brozzoli, Gentile, Petkova, \& Ehrsson, 2011; Cardini, et al., 2011; Gentile, Petkova, \& Ehrsson, 2011; Makin, Holmes, \& Zohary, 2007; Serino, Canzoneri, \& Avenanti, 2011).

It is largely accepted that the brain specialization for PPS has several functions in both animals and humans. These functions include the definition of the position of objects located near the body (Chieffi, Fogassi, Gallese, \& Gentilucci, 
1992; Moseley, Gallace, \& Spence, 2012) and the sustaining of a margin of safety around one's body (Graziano \& Cooke, 2006; Niedenthal, 2007). This understanding of the PPS suggests that its boundaries can be defined in two different ways, that is, using either a metric or a functional approach (Costantini, Ambrosini, Tieri, Sinigaglia, \& Committeri, 2010). According to the metric hypothesis, all the objects located within a given physical distance (e.g., 50-60 cm) from the body will fall into the PPS. Conversely, if the functional understanding of the PPS holds, PPS boundaries will dynamically change according to contingent factors. Currently, there seems to be a consensus reached that supports the functional hypothesis. Indeed, several studies have demonstrated that PPS boundaries can shrink or expand as a function of the properties of stimuli in the surrounding environment, for example, when the stimuli are approaching the body vs. receding it or being static (TajaduraJimenez, Valjamae, Asutay, \& Vastfjall, 2010), or when the stimuli have the capability to elicit emotional responses or not (Vagnoni, Lourenco, \& Longo, 2012).

Regarding the sensitivity of PPS boundaries to dynamic stimuli, it has been shown that PPS is more sensitive to approaching as compared to static objects. In this regard, Neuhoff and colleagues demonstrated that the terminal distance of approaching sound sources is underestimated (Neuhoff, Planisek, \& Seifritz, 2009). In the same vein, Serino and colleagues proposed a method for capturing the boundaries of PPS which involves using intensity-changing sounds and testing their influence on the detection of tactile stimuli due to audio-tactile interaction processes (Canzoneri, Magosso, \& Serino, 2012). In their study, participants responded to tactile stimuli delivered to the right hand at different delays from the onset of task-irrelevant intensity-changing sounds. These sounds, which were presented via a pair of loudspeakers placed near the hand, gave an impression of a 

Ferri et al.

moving sound source either approaching or receding from the participant's hand. Results showed that auditory stimuli speeded up the processing of a tactile stimulus at the hand as long as it was perceived at a limited distance from the hand, thus capturing the boundaries of PPS representation. This multisensory enhancement observed for sound sources inside the bounds of PPS was stronger for approaching than receding auditory stimuli, perhaps due to the larger biological salience of approaching stimuli (Tajadura-Jimenez, Valjamae, et al., 2010).

The impact of looming stimuli on PPS boundaries seems to be even stronger for emotion-inducing stimuli, as shown for threatening stimuli. This effect has been demonstrated by Vagnoni and colleagues in a behavioural study in which they used visual looming stimuli, either threatening or non-threatening (Vagnoni, et al., 2012). Participants were required to judge the time-to-collision for looming visual stimuli that expanded in size before disappearing. It was found that time-to-collision was underestimated for threatening stimuli (e.g., a spider) as compared to nonthreatening stimuli (e.g., a butterfly).

It is currently unknown whether auditory emotion-inducing looming stimuli, rather than visual, can similarly alter PPS boundaries. If we think on our everyday life, we can easily find examples suggesting that this is the case, especially, given the omnidirectional nature of spatial hearing. For instance, sounds of a growling dog are immediately perceived as threatening, and these are perceived even more threatening when the dog is running towards us and sounds are becoming louder (TajaduraJimenez, Valjamae, et al., 2010). In fact, we can react emotionally even when the dog is still far away and we are still not seeing it. This behaviour is likely to be paralleled by an alteration of the PPS boundaries. This example is in line with one of the functions ascribed to PPS, which can be referred to as defining a defence space 

Ferri et al.

(Cooke \& Graziano, 2004). According to this understanding of PPS function, its boundaries would change as the surrounding environment changes, i.e., whether there are perceived sources of threat or not.

From the perspective of PPS as "defence space" it is worth investigating whether approaching threatening sound stimuli will influence PPS boundaries. Experimental evidence supporting the hypothesis that negative looming sounds can shape PPS comes from two previous studies by Tajadura-Jiménez and colleagues (Tajadura-Jiménez, Pantelidou, Rebacz, Västfjäll, \& Tsakiris, 2011; TajaduraJimenez, Valjamae, et al., 2010). In a first study they showed that unpleasant approaching sound sources evoke more intense emotional responses than receding ones (Tajadura-Jimenez, Valjamae, et al., 2010) as revealed by electrodermal responses, electromyography and self-reported emotional experiences. This approaching-receding difference was found, however, only for negative emotioninducing sound sources and not for neutral or positive sounds. In a second study, Tajadura-Jiménez and colleagues (Tajadura-Jiménez, et al., 2011) investigated changes in personal space boundaries caused by listening to either positive or negative emotion-inducing music. In this study, personal space was defined as the comfort interpersonal distance between a participant and an experimenter approaching the participant. They found that, in contrast with the negative condition, listening to positive emotion-inducing music shrank the representation of the participants' personal space, thus allowing others to come closer to them. The study by TajaduraJiménez and colleagues, however, tested the impact of emotional auditory stimuli on personal space, as defined in social psychology, which not necessarily corresponds to the PPS, as defined in cognitive neuroscience. Moreover, the auditory stimuli in that 

Ferri et al.

study were only used to change the emotional context in which a different stimulus (i.e., the experimenter) approached the participant.

In the present study we investigated whether emotion-inducing looming sound sources affect PPS representation. In two experiments participants were exposed to artificial and ecological sounds simulating looming (i.e., approaching) sound sources. Rising intensity level simulated the approaching nature of sound sources. Previous research has shown that the most salient cue for auditory motion perception is intensity change (Lutfi \& Wang, 1999), and therefore, sounds rising in intensity are generally perceived as approaching sound sources (for similar procedures see: Maier \& Ghazanfar, 2007; Neuhoff, 2001; Rosenblum, Carello, \& Pastore, 1987; TajaduraJimenez, Valjamae, et al., 2010).

In a first experiment, participants performed a simple tactile detection task of stimuli presented to their right hand, while listening to concurrently presented task-irrelevant artificial sound sources approaching toward their body (a similar procedure was used in (Canzoneri, et al., 2012; Finisguerra, Canzoneri, Serino, Pozzo, \& Bassolino, 2014; Teneggi, Canzoneri, di Pellegrino, \& Serino, 2013). The spectral properties of the sounds induced affective responses of either neutral or negative emotional valence. In agreement with the perspective of PPS as "defence space, we expected larger PPS when the approaching sound stimulus was negative as compared to neutral. In Experiment 2, we used ecological sounds, which content (i.e., psychological associations to the sound producing source), rather than physical properties, elicited emotional responses of negative, positive or neutral valence. Again, according to the defence space perspective, we expected to find a larger PPS when the approaching stimulus was a negative, threatening sound as compared to neutral or positive. 

Ferri et al.

\section{Methods}

\subsection{Experiment 1}

\subsubsection{Participants}

Twenty healthy subjects (17 females, mean age 21 years, range: 18-23) participated in Experiment 1 and twenty-five ( 23 females, mean age 21 years, range: 18-23) in Experiment 2. All participants were right-handed and had normal hearing, as self- reported. All subjects (students at the University of Chieti) gave their written informed consent to participate in the study, which was approved by the Ethical Committee of University "G. d'Annunzio", and was performed in accordance with the Declaration of Helsinki.

\subsubsection{Artificial sounds selection and validation}

The experimental stimuli were various power-law shaped noises with flat or increasing ("looming") intensity levels of $3000 \mathrm{~ms}$ duration. In power-law noises the power spectral density (PSD) is changed according to the equation $1 / \mathrm{f}^{\beta}$, where $\beta$ can be $0,+/-1,+/-2$. Noise sounds were "white" (flat PSD), "pink" (PSD change of 1/f), "brown" (PSD change of 1/f2), "blue" (PSD change of $\mathrm{f}$ ), and "violet" (PSD change of $\mathrm{f}^{2}$ ). The noises were all equalized to have equal power in the range from 2700 to $3150 \mathrm{~Hz}$, which corresponds to the 16th and most sensitive frequency band according to the Bark scale (Zwicker, 1961) and ISO226 equal-loudness contours (ISO226:2003). The sounds were sampled at $44.1 \mathrm{kHz}$ and presented by means of headphones. Sounds were manipulated by using the Soundforge 4.5 software (Sonic Foundry, Madison, WI), so that they had either flat or exponentially rising acoustic intensity from 55 to $70 \mathrm{~dB}$. As previously mentioned, when rising in intensity, sounds give the impression of sources moving towards the participant's body (Canzoneri, et al., 2012; Teneggi, et al., 2013). 

Ferri et al.

In a pre-experimental stimuli validation session, a group of participants $(\mathrm{N}=$ $40,21$ Female, mean age $=23$ years, range $=20-28)$ was invited to listen to all noise sounds and rate the emotion elicited by the sounds using the Self-Assessment Manikin (SAM, Bradley \& Lang, 1994; Lang, 1980), a test widely used in emotion research which consists of two 9-point pictorial scales. One scale serves to rate the valence or pleasantness of emotional feelings, and depicts nine manikins ranging horizontally from happy (or positive) to unhappy (or negative); the other scale, serves to rate the arousal or excitement of emotional feelings, and depicts nine manikins ranging horizontally from excited (or aroused) to calm (or relaxed). This procedure allowed selecting and validating two artificial sounds for Experiment 1, one inducing negative emotional responses and one inducing neutral ones.

\subsubsection{Procedure}

Experimental stimuli were looming auditory stimulus lasting $3000 \mathrm{~ms}$. In agreement with the results from the pre-experimental stimuli validation study (see Results section) we selected the Brown and the White noises as the "negative" and the "neutral" experimental stimuli. Along with the auditory stimulus, in $85 \%$ of the trials subjects were also presented with a tactile stimulus, delivered by means of a current constant stimulator (Digitimer DS7A) via a pair of $\mathrm{Ag}-\mathrm{AgCl}$ surface electrodes placed on the intermediate phalange of the right middle finger. The electrical tactile stimulus was a single, constant voltage, square wave pulse of 100$\boldsymbol{\mu}$ sec duration. The remaining trials ( $15 \%$ out of total) were catch trials with auditory stimulation only. Before the experiment, the intensity of the tactile stimulus was set to be clearly above threshold. This threshold was set individually for each subject, as follows: the intensity of the stimulator was set at the minimum value and then progressively increased until the subject reported to clearly perceive the 

Ferri et al.

stimulation. Then, the subject was presented with a series of 10 stimuli, all at that level of stimulation, intermingled with 5 catch trials in which no stimulation was presented. The subject was, asked to report when he/she felt the tactile stimulus. If the subject did not perform $\mathbf{1 0 0 \%}$ correct (i.e., if he/she failed to respond to some stimuli or gave false positives to the catch trials), the intensity was further increased by a $5 \mathbf{m A}$ step, and the procedure was repeated.

Subjects were blindfolded and sat down with their right arm resting, palm down, on a table beside them. They were asked to press a button with their left index finger when a tactile target was delivered, trying to ignore the auditory stimulus. The presentation of the stimuli and the recording of participants' responses were controlled by a custom software (developed by Gaspare Galati at the Department of Psychology, Sapienza Universita` di Roma, Italy), and implemented in Matlab (The MathWorks Inc., Natick, MA, USA) using Cogent 2000 (developed at FIL and ICN, UCL, London, UK) and Cogent Graphics (developed by John Romaya at the LON, Wellcome Department of Imaging Neuroscience, UCL, London, UK).

Tactile stimuli were presented with different delays with respect to the onset of auditory stimuli. In particular, ten different delays (referred to as D1-D10) were used, ranging from $300 \mathrm{~ms}$ to $3000 \mathrm{~ms}$, in steps of $300 \mathrm{~ms}$. For each trial, the sound was preceded and followed by $1000 \mathrm{~ms}$ of silence. In this way, tactile stimulation occurred when the sound source was perceived at different locations with respect to the subject's body: i.e., close to the body at high temporal delays and far from the body at low temporal delays.

Finally, in order to measure RTs in the unimodal tactile conditions (without any sound), tactile stimulation could be also delivered during the silence periods, preceding sound administration, namely at $500 \mathrm{~ms}$ (D0) before the beginning of the 

Ferri et al.

sound. The total experiment consisted of a random combination of 16 target stimuli repetitions for each of the temporal delays (D0-D10), and for both the brown and white noise stimuli, resulting in a total of 352 trials with a tactile target, randomly intermingled with 64 catch trials with auditory stimulation only. Trials were equally divided into two experimental blocks.

\subsubsection{Perceived distance manipulation test: noise}

To verify that subjects actually perceived the sound source (brown or white noise) at different locations according to different temporal delays in our experimental setup, we ran a sound localization experiment on 16 naïve subjects (14 females, mean age 22 years, range: 18-28). Participants sat down with their right arm, resting palm, down on a table beside them. They received a tactile stimulation on the index finger at one of five possible temporal delays, namely D1, D3, D5, D7 and D9, in a random series of 80 trials. At the end of each trial, they were asked to verbally indicate the perceived position of the sound source (brown or white noise) in the surrounding space on a scale from 1 (very far) to 100 (very close) at the time when they had felt the tactile stimulus. Figure 1A clearly shows that, for both noises, subjects progressively perceived the sound source closer to their body according to the increasing temporal delay (from D1 to D9) of the onset of the tactile stimulus.

\subsubsection{Data analysis}

Mean RTs for tactile targets were calculated for every temporal delay, separately for each sound. Mean RTs to the tactile targets at the different temporal delays were fitted to a sigmoidal function as described in Canzoneri et al. (2012) using five temporal delays obtained by averaging contiguous temporal delays. This procedure was implemented to reduce variability of each observed point in the curve. Sigmoid function solves non-linear least squares problems and returns several parameters 

Ferri et al.

including the central point $\left(\mathrm{x}_{\mathrm{c}}\right)$, referring to the value of the abscissa at the central point of the sigmoid, and $b$, referring to the slope of the sigmoid at the central point. Following previous studies (Canzoneri, et al., 2012; Teneggi, et al., 2013), for each participant and each sound condition we took $x_{c}$ as an estimation of the boundaries of individual PPS representation, and $b$ as an indication of the sharpness of the transition between the far and the near space. To test for the extent of PPS representation, we ran paired sample t-tests between the $x_{c}$ in the different sound conditions.

In preliminary analyses we fitted our data using both a linear and a sigmoidal function to test which model fitted better our data. Results showed that all the sounds fitted better a sigmoid function than a linear function. This was further supported by statistical analyses. For each sound we compared the root mean square errors (RMSE) of the sigmoid and linear functions to test which function explained the variance better. All the t-tests showed better variance fit (i.e., lower RMSE) for the sigmoid than the linear function (See Table 1).

\section{[Insert Table 1 about here]}

\section{Experiment 2}

\subsubsection{Stimuli and procedure}

The experimental stimuli were three ecological looming sounds ('Woman Screaming'; 'Baby Laughing' and 'Brush Teeth') of $3000 \mathrm{~ms}$ duration. The three sounds have the capability of inducing emotional responses with negative, positive or neutral valence, respectively. They were selected from the International Affective Digitized Sounds (IADS) database (sounds numbers: 276, 110 and 720), based on their normative emotional ratings (Bradley \& Lang, 1999). The sounds were sampled at $44.1 \mathrm{kHz}$ and presented by means of headphones. Sounds were manipulated by 

Ferri et al.

using the Soundforge 4.5 software to have exponentially rising acoustic intensity from 55 to $70 \mathrm{~dB}$. In this way sounds gave the impression of being moving towards the participant's body. Along with the auditory stimulation, in $77 \%$ of trials subjects were also presented with a tactile stimulus, delivered as in Experiment 1. The remaining trials $(23 \%$ out of total) were catch trials with auditory stimulation only. The experimental procedure and the temporal delays between the onset of the sound and the tactile stimulation were the same as in Experiment 1 (i.e., 10 different delays from $300 \mathrm{~ms}$ to $3000 \mathrm{~ms}$, in steps of $300 \mathrm{~ms}$ ). In order to measure RTs in the unimodal tactile condition (without any sound), tactile stimulation could be also delivered during the silence periods, preceding sound administration, namely at 500 ms (D0) before the beginning of the sound.

The total experiment consisted of a random combination of 12 target stimuli repetitions for each temporal delay (D0-D10), for each of the four sounds, resulting in a total of 528 trials with a tactile target, randomly intermingled with 160 catch trials with auditory stimulation only. Trials were equally divided into four presentation blocks. Data were analyzed as described in subsection 2.1.5 of Experiment 1.

In order to check that the selected sounds induced the expected emotional effects, in a separate experiment we invited a subgroup of our participants $(\mathrm{N}=15)$ to listen to all sounds and rate their emotional feelings using SAM.

\subsubsection{Perceived distance manipulation test: ecological sounds}

As in Experiment 1, we tested whether subjects actually perceived the emotional sound sources (negative, neutral and positive) at different locations according to different temporal delays in our experimental setup. We ran this sound localization test on the same subjects as in the previous distance perception study (see section 

Ferri et al.

2.1.4). Participants sat down as previously described. They received a tactile stimulation on the index finger at one of five possible temporal delays, namely D1, D3, D5, D7 and D9 in a random series of 120 trials. At the end of each trial, they were asked to verbally indicate the perceived position of the sound source (negative, neutral or positive) in space when they had felt the tactile stimulus, on a scale from 1 (very far) to 100 (very close). Figure 1B clearly shows that, for all sounds, subjects progressively perceived the sound source closer to their body according to the increasing temporal delay (from D1 to D9) of the onset of the tactile stimulus.

\section{[Insert Figure 1 about here]}

\section{Results}

\subsection{Experiment 1}

\subsubsection{Noise stimuli emotional validation}

Self-reported valence and arousal of emotional reaction to the different noises (SAM ratings) were used as dependent variables for a $5 \times 2$ MANOVA containing 'noise colour' (blue, brown, pink, white, violet) and 'looming' (on/off) as within-participants factors. The results (Figure 1) revealed that there was a significant main effect of 'noise colour' $(\mathrm{F}(8,310)=4.04 ; \mathrm{p}<0.001, \Lambda=0.82)$ and a significant interaction between 'looming' and 'noise colour' $(F(8,310)=2.42$; $p<$ $0.05, \Lambda=0.89$ ). These effects were mainly due to the arousal ratings: there was a significant main effect of 'noise colour' $(F(1.7,67.9)=8.39 ; p<0.001)$ and a significant interaction effect $(\mathrm{F}(3.3,129.5)=3.11 ; \mathrm{p}<0.05)$. Newman-Keuls post-hoc comparisons showed several significant differences between the noise stimuli. For valence, three pairs of pink-brown, blue-violet and white-violet showed significant differences (all at $\mathrm{p}<0.05$, see Figure 2 for details). For arousal, the five pairs showed significant differences: pink-brown, brown-blue, brown-white, blue-violet 

Ferri et al.

and violet-white (all at $\mathrm{p}<0.05$ ). From these results, we could see that brown and pink noises were judged as more arousing and less pleasant compared to the white noise stimulus. Based on these results we selected the white noise as a "neutral" sound and the brown noise as a "negative" sound.

\section{[Insert Figure 2 about here]}

\subsubsection{Central Point and slope of the sigmoid functions}

Three participants were discarded from the analysis because their data did not fit either the sigmoid or the linear function. Their individual RMSE exceeded the mean group RMSE of 2 standard deviations for both fitting functions. A paired sample t-test was run to compare the extent of PPS representation, as defined by the central points of the sigmoid functions, in the negative and in the neutral sound conditions. The sigmoid central point was lower in the negative sound condition (1529 $\mathrm{ms} \pm 310 \mathrm{~ms}$, Figure 3$)$ as compared to the neutral sound condition (1731 ms \pm $201 \mathrm{~ms} ; \mathrm{t}(16)=-2.2 ; \mathrm{p}<0.041$, two-tailed), suggesting that PPS boundaries were farther from the participants when they were presented with the task irrelevant negative sound as compared to the task irrelevant neutral sound (see Figure 3). In addition, the slope at the central point tended to be steeper in the negative sound ($0.43 \pm 0.27)$ condition as compared to the neutral sound condition $(-0.23 \pm 0.24, \mathrm{t}(16)$ $=-1.9 ; \mathrm{p}=0.07$, two-tailed).

\section{[Insert Figure 3 about here]}

\subsection{Experiment 2}

\subsubsection{Ecological sounds emotional validation}

In order to assess the emotional effects of the ecological sounds, we asked a subgroup of our participants $(\mathrm{N}=15 ; 14$ females, mean age 23.5 years, range: $20-37)$ to rate their emotional reactions to the sounds as above. Self-reported valence and 

Ferri et al.

arousal SAM ratings were used as dependent variables for a 1-way MANOVA containing 'sound' (negative, neutral, positive, white noise) as within-participants factor. The results shown in Figure 4 revealed that there was a significant main effect of sound $(F(6,82)=8.82 ; p<0.001, \Lambda=.37)$, for both valence $(F(2.12,29.63)$ $=15.09 ; p<0.001)$ and arousal dimensions $(F(2.37,33.12)=5.68 ; \mathrm{p}<0.01)$. The 'negative' sound was rated as more unpleasant than either of the other sounds (neutral: $\mathrm{t}(14)=3.38, \mathrm{p}<0.01$; positive: $\mathrm{t}(14)=5.98, \mathrm{p}<0.001$; white: $\mathrm{t}(14)=2.19$, $\mathrm{p}$ $<0.05$ ), as well as more arousing than either of the other sounds (neutral: $t(14)=6.52$, $\mathrm{p}<0.001$; positive: $\mathrm{t}(14)=2.82, \mathrm{p}<0.05$; white: $\mathrm{t}(14)=3.35, \mathrm{p}<0.01)$. The 'positive' sound was rated as more pleasant than the neutral and the white sounds (neutral: $\mathrm{t}(14)=4.21, \mathrm{p}<0.01$; white: $\mathrm{t}(14)=4.63, \mathrm{p}<0.01$ ), but not more arousing than them (all ps $>0.39$ ). As expected, the neutral sound did not differ in pleasantness or arousal from the white noise (all ps $>0.72$ ), thus validating the choice of the "neutral" sound as "neutral", equivalent to the sound used in Experiment 1. For this reason the white noise was not longer considered during the data analysis.

\section{[Insert Figure 4 about here]}

\subsubsection{Central point and slope of the sigmoid functions}

Three participants were discarded from the analysis because their data did not fit either the sigmoid or the linear function. Their individual RMSE was, indeed, higher than 2 standard deviations from the group RMSE in both the fitting functions. Paired sample t-tests were run to compare the extent of PPS representation, as defined by the central points of the sigmoid functions, in the different sound conditions. The sigmoid central point was lower in the negative sound condition (1325 $\mathrm{ms} \pm 434 \mathrm{~ms}$; Figure 5, solid line) as compared to the neutral (1496 ms \pm 521 ms; Figure 5, dashed line; $\mathrm{t}(21)=-2.60 ; \mathrm{p}=0.02$, two-tailed) and positive sound 

Ferri et al.

conditions (1641 ms $\pm 483 \mathrm{~ms}$; Figure 5, dotted line; $\mathrm{t}(21)=-3.10 ; \mathrm{p}<0.007$, twotailed), suggesting that PPS boundaries were farther from the participants when they were presented with a task irrelevant negative sound as compared to either a neutral and a positive sound (see Figure 5). No significant effects were found in the analysis of the slopes (Negative sound: $-0.42 \pm 0.30$; Neutral sound: $-0.22 \pm 0.36$; Positive sound: $-0.21 \pm 0.30)$.

\section{[Insert Figure 5 about here]}

\section{Discussion}

We investigated whether emotion-inducing looming sound sources, as compared to neutral looming sound sources, have an impact on the boundaries of PPS representation. We expected changes in the size of the comfort/safety zone around one's body as a result of the emotional saliency of the sound sources approaching it. To this aim, we used a well-established multisensory task (Canzoneri, et al., 2012; Teneggi, et al., 2013) allowing virtually demarcating the boundary of the PPS representation.

In two experiments, participants were exposed to either artificial (Experiment 1) or ecological (Experiment 2) sounds, which simulated, by changes in their intensity, the rapid approach of a sound source towards the participant's body. Only emotion-inducing sounds that were unambiguously classified as positive, negative or neutral were included as stimuli in our study.

In Experiment 1 looming stimuli were artificial sounds (noises) that induced emotional responses with either neutral or negative emotional valence, depending on the physical sound properties, specifically, their spectral density characteristics. Results from this experiment showed that the boundary of PPS was "located" at around $1731( \pm 201)$ ms after the onset of the neutral sound and at around 

Ferri et al.

$1529( \pm 310) \mathrm{ms}$ after the onset of the negative sound (see Figure 3). That is, negative sounds evoked a larger PPS as compared to neutral sounds.

In Experiment 2 looming stimuli were ecological sounds (from the IADS database) that induced emotional responses with neutral, negative or positive emotional valence. The valence of the emotional responses induced by these sounds mostly depended on the semantic content of the sound. Thus, this experiment looked at whether the effect of negative valence observed in Experiment 1 can be generalized to other sounds found in a natural setting (Ho, Santangelo, \& Spence, 2009; Ho \& Spence, 2008). Importantly, differently from Experiment 1, here we also looked at whether the effect on the PPS representation boundary is specific to negative valence or whether it applies to other emotion-inducing sounds of different valence (i.e., sounds inducing positive emotional responses). In agreement with Experiment 1, the results showed that the boundaries of PPS were "located" at 1496 $( \pm 521) \mathrm{ms}$ after the onset of the neutral sound, while they were located at $1325( \pm 434)$ ms after the onset of the negative sound. The results from our Experiment 2 deepen those from Experiment 1 and clearly suggest that the valence of the emotional responses induced by approaching sound sources shapes the boundaries of PPS. Indeed, Experiment 2 further shows that positive sounds exert an opposite effect on the boundaries of the PPS representation than negative sounds. The results showed that these boundaries shrank after the onset of a positive sound (they were "located" at $1641( \pm 483) \mathrm{ms}$ after the onset of the positive sound).

We did not find any significant differences between the slopes of the sigmoidal functions in neither of the experiments. This negative finding might be taken as evidence that the emotional valence of the approaching stimulus influences the boundaries of PPS without impacting on the sharpness of the transition between 

Ferri et al.

the PPS and the extrapersonal space (Van der Stoep, Nijboer, Van der Stigchel, \& Spence, 2014).

Possibly, one may argue that our results are at odds with some previous findings. Teneggi and colleagues (2013), using the same paradigm we employed in the current study, showed that fair cooperative interaction with another person brought to an expansion of PPS. Here, the expansion of PPS (i.e., the same directional change in the PPS boundary) is observed with negative valence attributed to the looming sound (Experiments 1 and 2). These apparently conflicting results can be reconciled if interpreted in the light of the different functions of PPS recently described by De Vignemont and Ianetti (2014). They proposed a distinction between PPS functions defining a "protective space" and a "working space". The former functional role would be protection of the body, while the latter would serve goal-directed actions. Moreover, according to the same authors, joint action does not require a third kind of PPS function, distinct from the working and the protective space. Indeed, fair cooperative action is clearly related to the working space, whereas potentially harmful joint action is related to the protective space (de Vignemont \& Iannetti, 2014). Which PPS function is involved in our study as compared to Teneggi et al.'s study? We suggest that the negative sounds we employed elicit expansion of PPS as protective space. Differently, in Teneggi et al' $s$ study the fair cooperation induces expansion of PPS as working space, because the cooperative partner needs to be included in one's (shared) PPS. In sum, the same outcome (i.e., expansion of PPS) is elicited by opposite contexts (i.e., negative sounds vs. friendly cooperation) here and in Teneggi et al.'s (2013) as the targeted PPS function is different in the two studies. 

Ferri et al.

It is important to note here that we observed modulations of the boundary of PPS representation by auditory-induced emotion despite the fact that both sounds and their emotional content were irrelevant to the task. How can we account for this effect? We suggest that this effect could be explained by the relationship between emotional sound processing and PPS representation. Our perceptual systems are responsible for informing us about the environment and for keeping a constant spatial margin of safety surrounding our body. Thus, they are in charge of continuously monitoring the nearby space in order to alert us of any significant events requiring an action from our side (Graziano, 2001). In this respect, the auditory system, in particular, has a number of advantages over other sensory systems suggesting that its most basic function is to act as a warning system (e.g., Juslin \& Vastfjall, 2008). First, the auditory system provides us with a continuous stream of information since our ears cannot be "turned off" in the same way as we regularly block vision by closing our eyes (Larsson, 2005). Second, the auditory system has been characterized as a change detector that responds to certain sound properties indicating a rapid change by quickly orienting behaviour towards potential threats (Juslin \& Vastfjall, 2008). This is done in a faster way than the visual system does (McDonald, Teder-Salejarvi, \& Hillyard, 2000). The auditory system also complements the visual system by providing information about the events occurring outside one's visual field. With spatial hearing we can sense, without the need of turning our heads, both direct sounds emitted by different sources and their reflections from all directions in space. These reflections provide an impression of the geometry and size of the space we are in (see (Larsson, Väljamäe, Västfjäll, Tajadura-Jiménez, \& Kleiner, 2010). 

Ferri et al.

Keeping a constant margin of safety surrounding our body is also one of the main functions stemming from PPS representation (Graziano \& Cooke, 2006; Niedenthal, 2007), and in fact, the pivotal role of auditory stimuli in shaping multisensory PPS has been shown by several neurophysiological (Graziano \& Cooke, 2006; Graziano, Reiss, \& Gross, 1999), neuropsychological (Farnè \& Ladavas, 2002), and psychophysical studies (Kitagawa, Zampini, \& Spence, 2005; Tajadura-Jimenez, et al., 2009; Zampini, Torresan, Spence, \& Murray, 2007). For instance, Farnè and Ladavas (2002) investigated crossmodal audio-tactile extinction in eighteen rightbrain damaged patients. Tactile stimuli were delivered on the neck while auditory stimuli were delivered either near or far from the head. Results showed that only near auditory stimuli strongly extinguished contralesional tactile stimuli. This holds true when auditory stimuli were delivered in both the front or rear space.

The relevance of auditory stimuli in shaping multisensory PPS is supported also by neuroimaging studies. There is evidence showing that approaching sounds (tones rising in intensity level), recruit a distributed neural network subserving space recognition (Seifritz, et al., 2002), including the motor and premotor cortices, the intraparietal sulcus as well as the amygdala (Bach, et al., 2008). The amygdala has been described as a warning area (Bach, et al., 2008) and as a detector of relevant events in the environment (Sander \& Scheich, 2001). Neuroimaging and lesion studies have established an important role of the amygdala for the processing of complex auditory emotional signals, such as laughing and crying voices (Sander \& Scheich, 2001; Seifritz, et al., 2003), and fearful and angry voices, compared to neutral ones (Klinge, Roder, \& Buchel, 2010). Furthermore, the amygdala seems to play also a pivotal role in the definition of the space around the body. In a seminal study, Kennedy and colleagues (Kennedy, Glascher, Tyszka, \& Adolphs, 2009) 

Ferri et al.

reported the case of a patient with a complete amygdala lesion who was lacking any sense of personal space. This result has been corroborated by an imaging study showing activation of the amygdala related to close personal proximity (Kennedy, et al., 2009). It is thus possible to hypothesize that these amygdala centred distributed neural networks were recruited during our study.

From a more phenomenal perspective, a relation between emotional processing and PPS representation is in line with the considerable amount of behavioural evidence showing that the boundaries of PPS representation can be modulated by a multiplicity of factors, such as the characteristics of the stimulus (e.g., dynamic vs. static; emotionally laden vs. neutral) (Canzoneri, et al., 2012), the characteristics of the contextual environment (e.g., social vs. non-social, safety vs. threatening, (Tajadura-Jimenez, Larsson, Valjamae, Vastfjall, \& Kleiner, 2010; Tajadura-Jiménez, et al., 2011; Teneggi, et al., 2013) and individual personality traits (Sambo \& Iannetti, 2013; Vagnoni, et al., 2012). For instance, Lourenco and colleagues (Lourenco, Longo, \& Pathman, 2011) investigated whether the extension of PPS relates to individual differences in claustrophobic fear, defined as the fear of having no escape and being in closed or small spaces/rooms. They found trait feelings of claustrophobic fear predicting the size of near (PPS) space. Specifically, people with larger PPS reported higher rates of claustrophobic fear than people with smaller PPS. In the same vein, individuals with high scores on trait anxiety show larger PPS than individual with low trait anxiety scores (Sambo \& Iannetti, 2013).

Another example about how individual characteristics shape the boundary of PPS comes from a recent study investigating the rear PPS in two groups of non-clinical participants, categorized according to their sensitivity to cynophobia (Taffou \& Viaud-Delmon, 2014). The authors employ the same 

Ferri et al.

paradigm as here, with looming sounds approaching from the rear hemifield being either threatening (i.e., dog growling) or non- threatening auditory stimuli (i.e., sheep bleating). While in this study no differences in PPS size between threatening and non-threatening sound conditions were found for the no fearful participants, a difference in PPS size between the two sound conditions emerged for the participants fearful of dogs. These results thus demonstrate that cynophobic fear extends rear PPS in the presence of looming dog sounds, thus providing further support for our results showing that sound sources perceived as threatening extend PPS. Critically, our results for sound sources approaching from the frontal hemifield show a modulation of PPS size for the overall group of participants, independently of their fear level to the negative sound source, and show also the opposite effect in PPS size for sound sources identified as positive. Overall, in order to survive in a complex environment, inhabited by potentially threatening and noxious objects or living beings, as well as other individuals, first of all we need to constantly monitor the space immediately around our body. Such monitoring cannot rely on purely visual, auditory and/or emotional information. Our brain should simultaneously monitor and integrate all this information. The multisensory dynamic representation of PPS seems to be the best candidate for such processing.

\section{Acknowledgments}

We thank Andrea Serino and Elisa Canzoneri for their helpful suggestions. M.C. and F.F. were supported by a grant from the BIAL foundation ("The body beyond the body", Ref. 41/12). M.C. and A.T-J were supported by a grant from the Volkswagen Foundation's European Platform for Life Sciences, Mind Sciences and the 

Ferri et al.

Humanities ("The (Un)bound Body project. Exploring the constraints of embodiment $\&$ the limits of body representation", Ref. 85 639). A.T-J. was also supported by the ESRC grant ES/K001477/1 ("The hearing body"). A.V. was supported by Marie Curie Actions of the European Union's Seventh Framework Programme (FP7/20072013) under REA GA-303172. 


\section{References}

Avillac, M., Deneve, S., Olivier, E., Pouget, A., \& Duhamel, J. R. (2005). Reference frames for representing visual and tactile locations in parietal cortex. Nat Neurosci, 8, 941-949.

Bach, D. R., Schachinger, H., Neuhoff, J. G., Esposito, F., Di Salle, F., Lehmann, C., Herdener, M., Scheffler, K., \& Seifritz, E. (2008). Rising sound intensity: an intrinsic warning cue activating the amygdala. Cereb Cortex, 18, 145-150.

Bradley, M. M., \& Lang, P. J. (1994). Measuring emotion: the Self-Assessment Manikin and the Semantic Differential. J Behav Ther Exp Psychiatry, 25, 4959.

Bradley, M. M., \& Lang, P. J. (1999). International affective digitized sounds (IADS): Stimuli, instruction manual and affective ratings (Tech. Rep. No. B-2). Gainesville, FL: The Center for Research in Psychophysiology, University of Florida., (Chapter Chapter).

Bremmer, F., Schlack, A., Shah, N. J., Zafiris, O., Kubischik, M., Hoffmann, K., Zilles, K., \& Fink, G. R. (2001). Polymodal motion processing in posterior parietal and premotor cortex: a human fMRI study strongly implies equivalencies between humans and monkeys. Neuron, 29, 287-296.

Brozzoli, C., Gentile, G., Petkova, V. I., \& Ehrsson, H. H. (2011). fMRI Adaptation Reveals a Cortical Mechanism for the Coding of Space Near the Hand. The Journal of Neuroscience, 31, 9023-9031.

Canzoneri, E., Magosso, E., \& Serino, A. (2012). Dynamic sounds capture the boundaries of peripersonal space representation in humans. PLoS ONE, 7, e44306. 
Cardini, F., Costantini, M., Galati, G., Romani, G. L., Ladavas, E., \& Serino, A. (2011). Viewing one's own face being touched modulates tactile perception: an fMRI study. J Cogn Neurosci, 23, 503-513.

Chieffi, S., Fogassi, L., Gallese, V., \& Gentilucci, M. (1992). Prehension movements directed to approaching objects: influence of stimulus velocity on the transport and the grasp components. Neuropsychologia, 30, 877-897.

Cooke, D. F., \& Graziano, M. S. (2004). Sensorimotor integration in the precentral gyrus: polysensory neurons and defensive movements. J Neurophysiol, 91, $1648-1660$.

Costantini, M., Ambrosini, E., Tieri, G., Sinigaglia, C., \& Committeri, G. (2010). Where does an object trigger an action? An investigation about affordances in space. Experimental Brain Research, 207, 95.

de Vignemont, F., \& Iannetti, G. D. (2014). How many peripersonal spaces? Neuropsychologia.

Duhamel, J. R., Bremmer, F., Ben Hamed, S., \& Graf, W. (1997). Spatial invariance of visual receptive fields in parietal cortex neurons. Nature, 389, 845-848.

Farnè, A., \& Ladavas, E. (2002). Auditory peripersonal space in humans. J Cogn Neurosci, 14, 1030-1043.

Finisguerra, A., Canzoneri, E., Serino, A., Pozzo, T., \& Bassolino, M. (2014). Moving sounds within the peripersonal space modulate the motor system. Neuropsychologia.

Gentile, G., Petkova, V. I., \& Ehrsson, H. H. (2011). Integration of visual and tactile signals from the hand in the human brain: an FMRI study. J Neurophysiol, 105, 910-922. 
Graziano, M. S. (2001). A system of multimodal areas in the primate brain. Neuron, 29, 4-6.

Graziano, M. S., \& Cooke, D. F. (2006). Parieto-frontal interactions, personal space, and defensive behavior. Neuropsychologia, 44, 2621-2635.

Graziano, M. S., \& Gandhi, S. (2000). Location of the polysensory zone in the precentral gyrus of anesthetized monkeys. Exp Brain Res, 135, 259-266.

Graziano, M. S., \& Gross, C. G. (1993). A bimodal map of space: somatosensory receptive fields in the macaque putamen with corresponding visual receptive fields. Exp Brain Res, 97, 96-109.

Graziano, M. S., Reiss, L. A., \& Gross, C. G. (1999). A neuronal representation of the location of nearby sounds. Nature, 397, 428-430.

Hayduk, L. A. (1983). Personal space: Where we now stand. Psychol Bull, 94, 293 335.

Ho, C., Santangelo, V., \& Spence, C. (2009). Multisensory warning signals: when spatial correspondence matters. Exp Brain Res, 195, 261-272.

Ho, C., \& Spence, C. (2008). The Multisensory Driver: Implications for Ergonomic Car Interface Design. . Aldershot: Ashgate Publishing, (Chapter Chapter).

ISO226:2003. International Organization for Standardization. Acoustics-normal equal-loudness-level contours.

Juslin, P. N., \& Vastfjall, D. (2008). Emotional responses to music: the need to consider underlying mechanisms. Behav Brain Sci, 31, 559-575; discussion $575-621$.

Kennedy, D. P., Glascher, J., Tyszka, J. M., \& Adolphs, R. (2009). Personal space regulation by the human amygdala. Nat Neurosci, 12, 1226-1227. 
Kitagawa, N., Zampini, M., \& Spence, C. (2005). Audiotactile interactions in near and far space. Exp Brain Res, 166, 528-537.

Klinge, C., Roder, B., \& Buchel, C. (2010). Increased amygdala activation to emotional auditory stimuli in the blind. Brain, 133, 1729-1736.

Lang, P. J. (1980). Behavioral treatment and bio-behavioral assessment: computer applications. Norwood, NJ: Ablex.: In J. B. Sidowski, J. H. Johnson, \& T. A. Williams (Eds.) Technology in mental health care delivery systems, (Chapter Chapter).

Larsson, P., Väljamäe, A., Västfjäll, D., Tajadura-Jiménez, A., \& Kleiner, M. (2010). Auditory-Induced Presence in Mixed Reality Environments and Related Technology. In E. Dubois, P. Gray \& L. Nigay (Eds.), The Engineering of Mixed Reality Systems (pp. 143-163): Springer London.

Lourenco, S. F., Longo, M. R., \& Pathman, T. (2011). Near space and its relation to claustrophobic fear. Cognition, 119, 448-453.

Lutfi, R. A., \& Wang, W. (1999). Correlational analysis of acoustic cues for the discrimination of auditory motion. J Acoust Soc Am, 106, 919-928.

Maier, J. X., \& Ghazanfar, A. A. (2007). Looming biases in monkey auditory cortex. J Neurosci, 27, 4093-4100.

Makin, T. R., Holmes, N. P., \& Zohary, E. (2007). Is That Near My Hand? Multisensory Representation of Peripersonal Space in Human Intraparietal Sulcus. The Journal of Neuroscience, 27, 731-740.

McDonald, J. J., Teder-Salejarvi, W. A., \& Hillyard, S. A. (2000). Involuntary orienting to sound improves visual perception. Nature, 407, 906-908. 
Moseley, G. L., Gallace, A., \& Spence, C. (2012). Bodily illusions in health and disease: physiological and clinical perspectives and the concept of a cortical 'body matrix'. Neurosci Biobehav Rev, 36, 34-46.

Neuhoff, J. G. (2001). An Adaptive Bias in the Perception of Looming Auditory Motion. Ecological Psychology, 13, 87-110.

Neuhoff, J. G., Planisek, R., \& Seifritz, E. (2009). Adaptive sex differences in auditory motion perception: looming sounds are special. J Exp Psychol Hum Percept Perform, 35, 225-234.

Niedenthal, P. M. (2007). Embodying Emotion. Science, 316, 1002-1005.

Rizzolatti, G., Fadiga, L., Fogassi, L., \& Gallese, V. (1997). The space around us. Science, 277, 190-191.

Rizzolatti, G., Scandolara, C., Matelli, M., \& Gentilucci, M. (1981). Afferent properties of periarcuate neurons in macaque monkeys. II. Visual responses. Behavioural Brain Research, 2, 147-163.

Rosenblum, L. D., Carello, C., \& Pastore, R. E. (1987). Relative effectiveness of three stimulus variables for locating a moving sound source. Perception, 16, 175186.

Sambo, C. F., \& Iannetti, G. D. (2013). Better safe than sorry? The safety margin surrounding the body is increased by anxiety. J Neurosci, 33, 14225-14230.

Sander, K., \& Scheich, H. (2001). Auditory perception of laughing and crying activates human amygdala regardless of attentional state. Brain Res Cogn Brain Res, 12, 181-198.

Seifritz, E., Esposito, F., Neuhoff, J. G., Luthi, A., Mustovic, H., Dammann, G., von Bardeleben, U., Radue, E. W., Cirillo, S., Tedeschi, G., \& Di Salle, F. (2003). 
Differential sex-independent amygdala response to infant crying and laughing in parents versus nonparents. Biol Psychiatry, 54, 1367-1375.

Seifritz, E., Neuhoff, J. G., Bilecen, D., Scheffler, K., Mustovic, H., Schachinger, H., Elefante, R., \& Di Salle, F. (2002). Neural processing of auditory looming in the human brain. Curr Biol, 12, 2147-2151.

Serino, A., Canzoneri, E., \& Avenanti, A. (2011). Fronto-parietal Areas Necessary for a Multisensory Representation of Peripersonal Space in Humans: An rTMS Study. Journal of Cognitive Neuroscience, 23, 2956.

Sommer, R. (1959). Studies in Personal Space. Sociometry, 22, 247-260.

Taffou, M., \& Viaud-Delmon, I. (2014). Cynophobic fear adaptively extends peripersonal space. Front Psychiatry, 5, 122.

Tajadura-Jimenez, A., Kitagawa, N., Valjamae, A., Zampini, M., Murray, M. M., \& Spence, C. (2009). Auditory-somatosensory multisensory interactions are spatially modulated by stimulated body surface and acoustic spectra. Neuropsychologia, 47, 195-203.

Tajadura-Jimenez, A., Larsson, P., Valjamae, A., Vastfjall, D., \& Kleiner, M. (2010). When room size matters: acoustic influences on emotional responses to sounds. Emotion, 10, 416-422.

Tajadura-Jiménez, A., Pantelidou, G., Rebacz, P., Västfjäll, D., \& Tsakiris, M. (2011). I-Space: The Effects of Emotional Valence and Source of Music on Interpersonal Distance. PLOS ONE, 6, e26083.

Tajadura-Jimenez, A., Valjamae, A., Asutay, E., \& Vastfjall, D. (2010). Embodied auditory perception: the emotional impact of approaching and receding sound sources. Emotion, 10, 216-229. 
Teneggi, C., Canzoneri, E., di Pellegrino, G., \& Serino, A. (2013). Social modulation of peripersonal space boundaries. Curr Biol, 23, 406-411.

Vagnoni, E., Lourenco, S. F., \& Longo, M. R. (2012). Threat modulates perception of looming visual stimuli. Curr Biol, 22, R826-827.

Van der Stoep, N., Nijboer, T. C., Van der Stigchel, S., \& Spence, C. (2014). Multisensory interactions in the depth plane in front and rear space: A review. Neuropsychologia.

Zampini, M., Torresan, D., Spence, C., \& Murray, M. M. (2007). Auditorysomatosensory multisensory interactions in front and rear space. Neuropsychologia, 45, 1869-1877.

Zwicker, E. (1961). Subdivision of the Audible Frequency Range into Critical Bands(Frequenzgruppen). J Acoust Soc Am, 33, 248-248. 


\section{FIGURE CAPTIONS:}

Table 1: Root mean square error of the sigmoidal and linear functions in all the experimental conditions.

Figure 1. Results of the sound localization experiments. Panel A refers to experiment 1, panel B refers to experiment 2.

Figure 2. Results of the stimuli validation study for Experiment 1. Mean valence and arousal ratings (in a 9-point scale) for all noise sounds. Circle: Looming sounds; Diamond: Flat sounds; P: Pink noise; BR: Brown noise; W: White noise; V: Violet noise; B: Blue noise. Error bars indicate the standard error of the means.

Figure 3. Best-fitting sigmoidal functions describing the relationship between RTs and sound distance in the Negative sound condition (Solid sigmoid) and the Neutral sound condition (dashed sigmoid). The solid and the dashed vertical lines represent the central point of the negative and neutral sounds, respectively.

Figure 4. Results of the stimuli validation study for Experiment 2. Mean valence and arousal ratings (in a 9-point scale) for all sound conditions (positive, negative, neutral and white). Error bars indicate the standard error of the means.

Figure 5. Best-fitting sigmoidal functions describing the relationship between RTs and sound distance in the Negative sound condition (Solid sigmoid), the Neutral sound condition (dashed sigmoid) and the Positive sound condition (dotted sigmoid). The solid, dashed and dotted vertical lines represent the central point of the negative, neutral and positive sounds, respectively. 


\begin{tabular}{lccc}
\hline Experiment 1 & RMSE - Sigmoid & RMSE - Linear & p \\
\hline Brown & 47 & 63 & 0.005 \\
White & 46 & 61 & 0.027 \\
Experiment 2 & & & \\
Negative & 63 & 104 & 0.028 \\
Neutral & 78 & 115 & 0.015 \\
Positive & 66 & 107 & 0.068 \\
\hline
\end{tabular}

Table 1: root mean square errors for the sigmoid and linear function. 


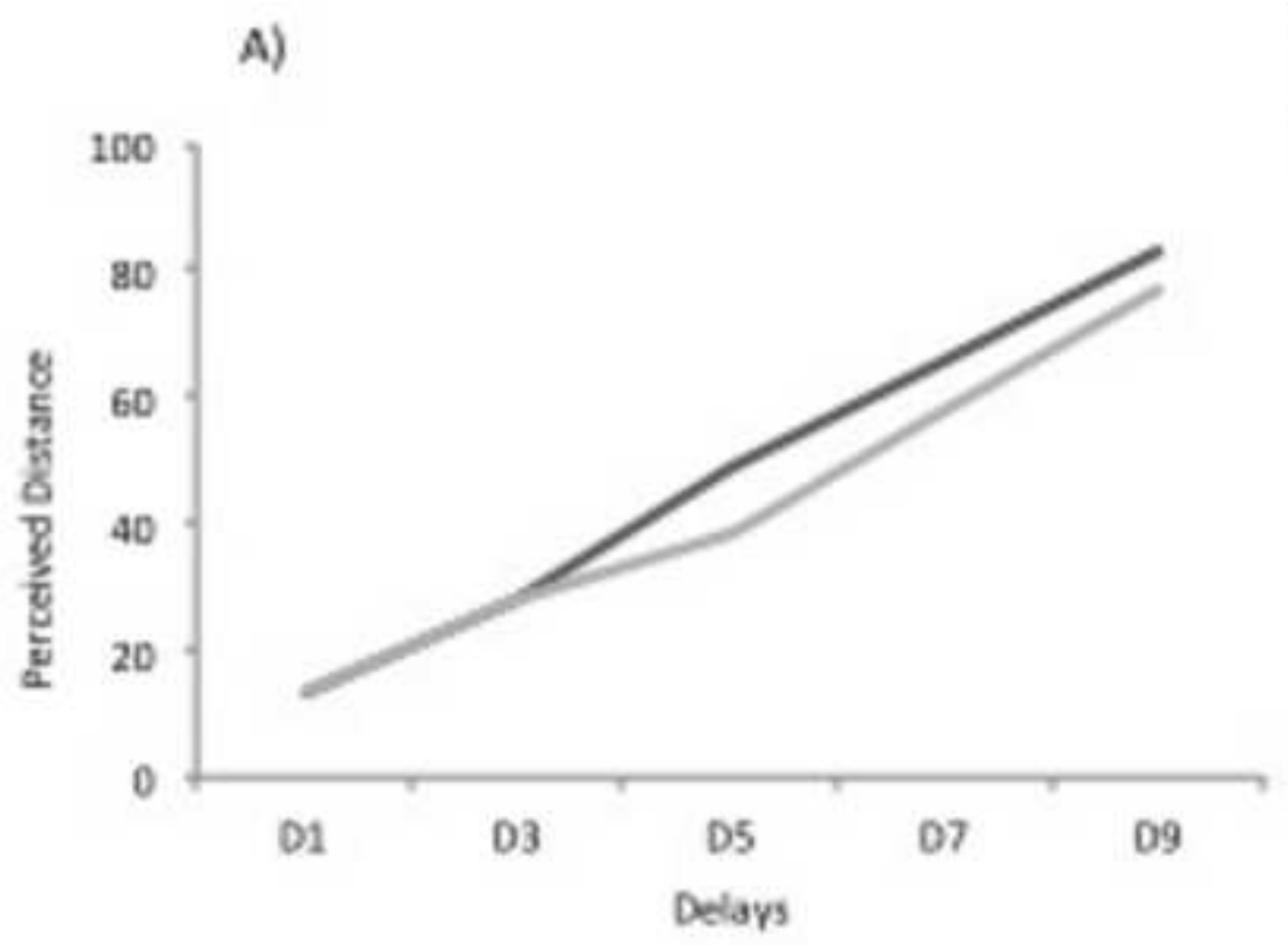

B)
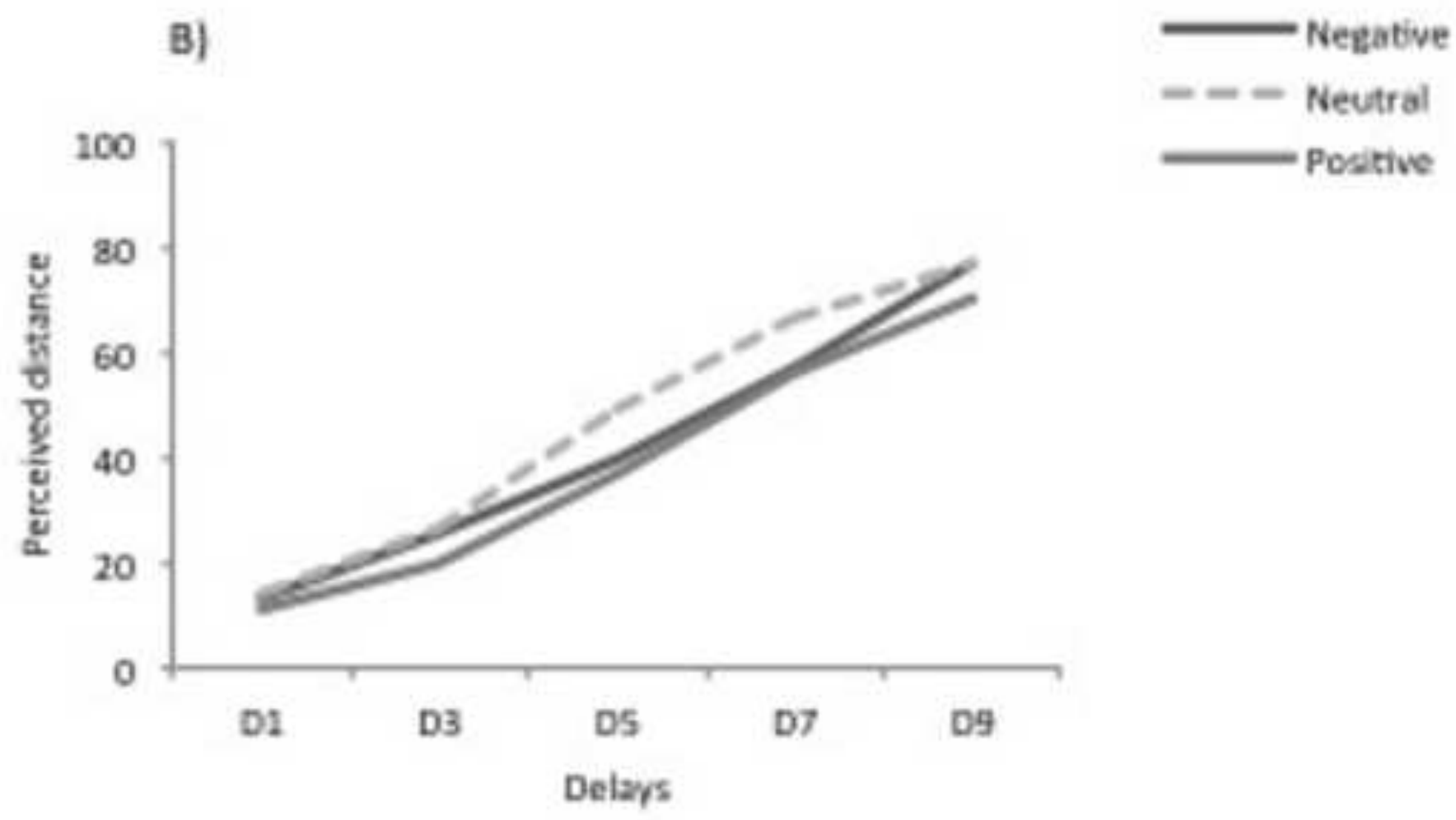


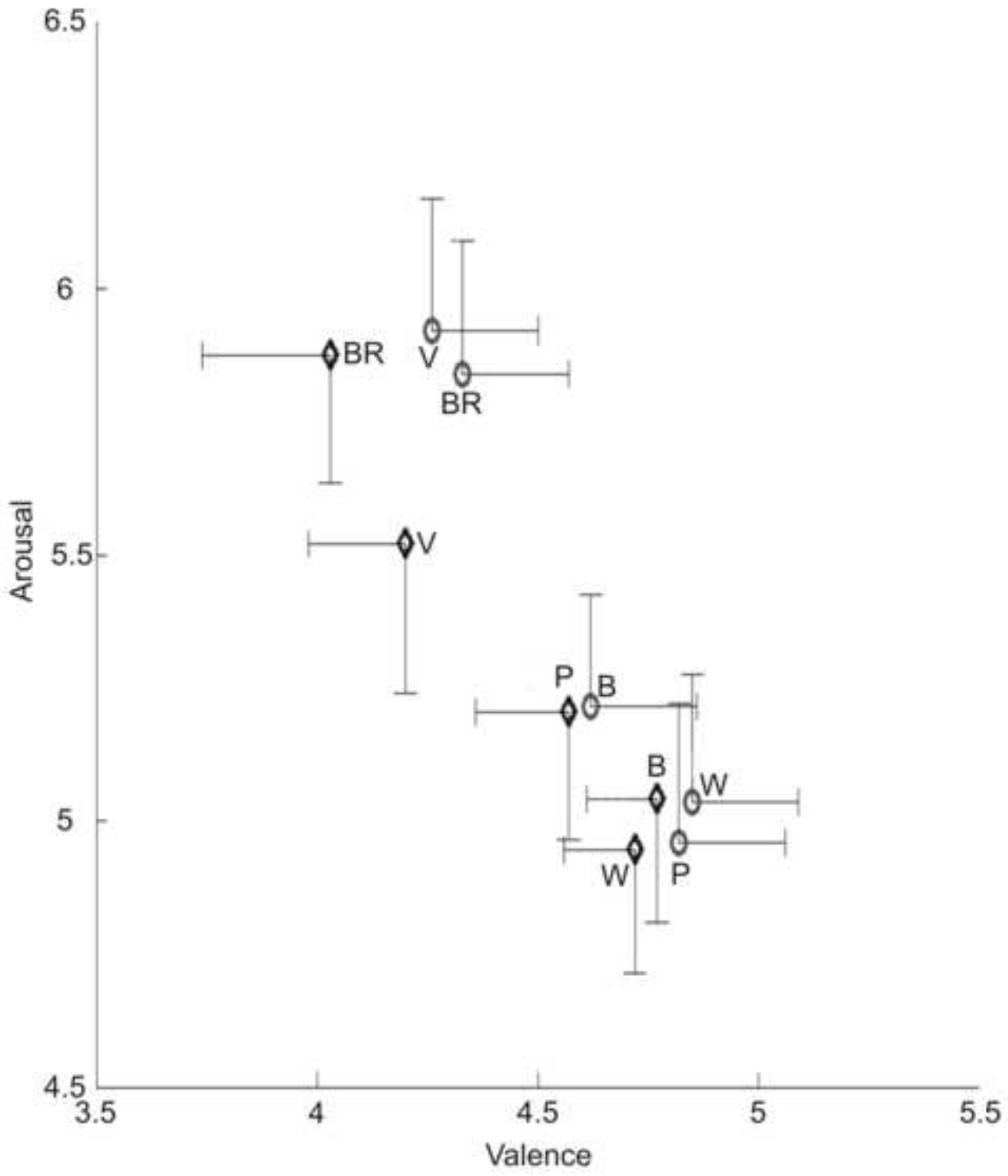




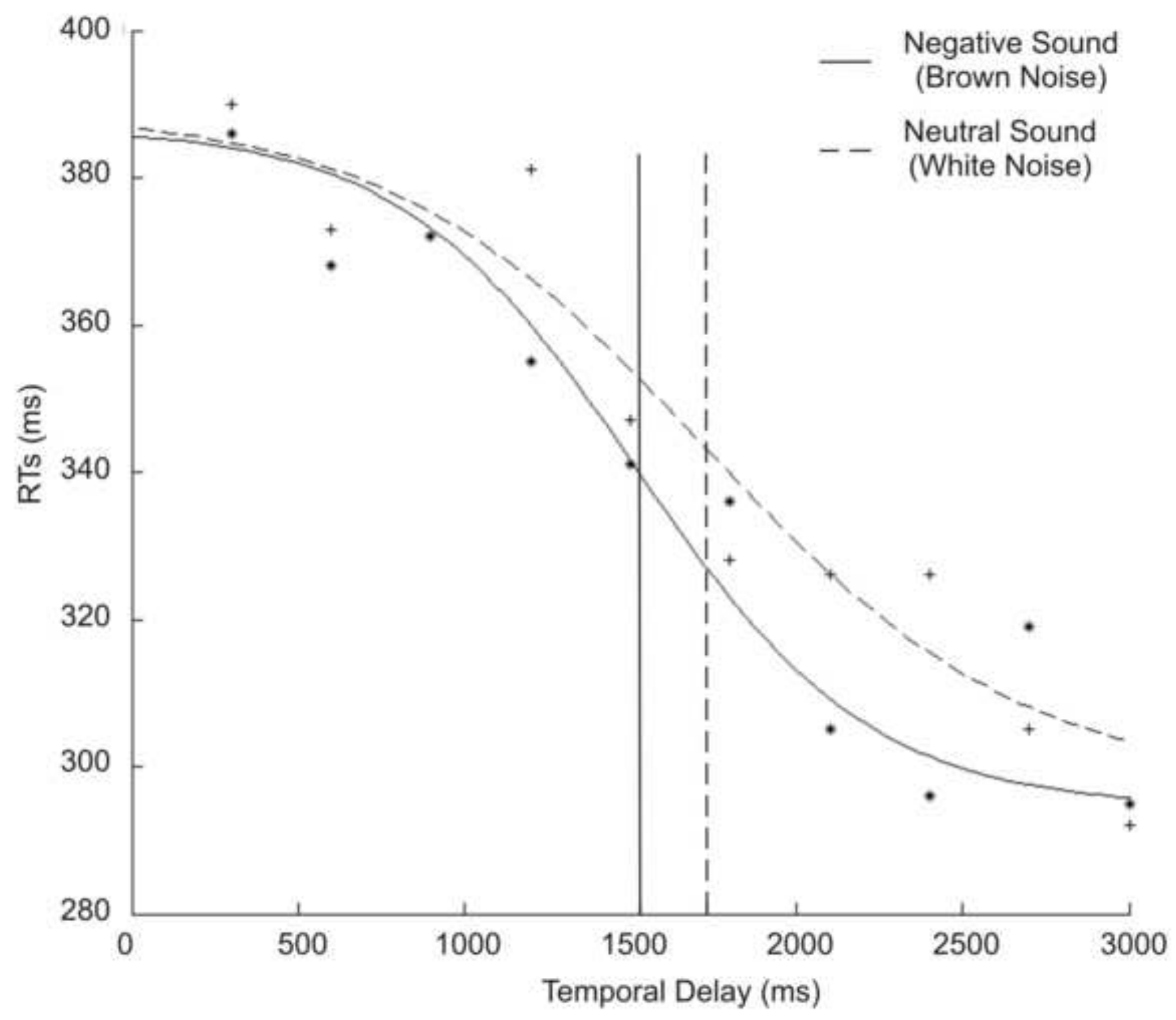




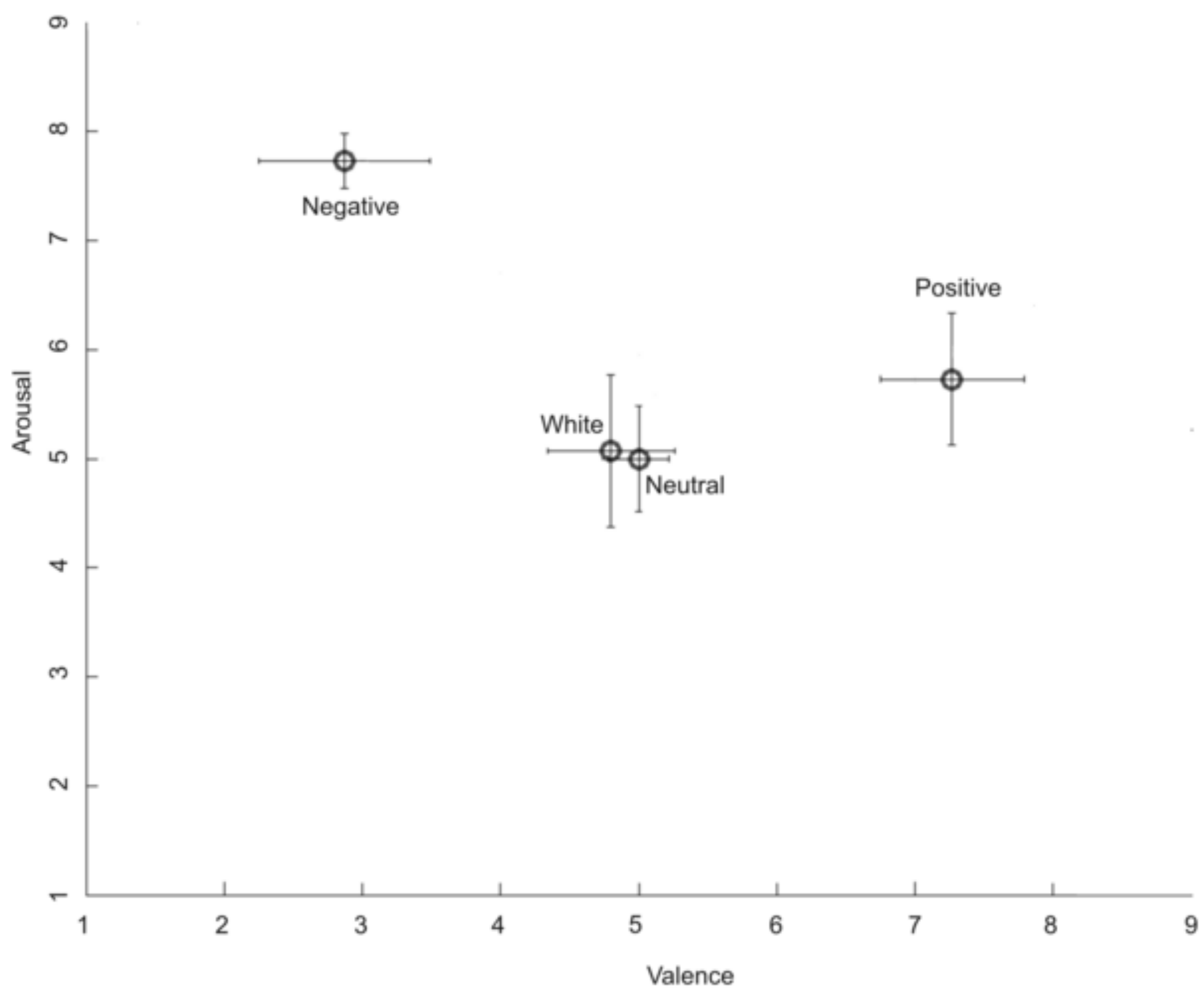




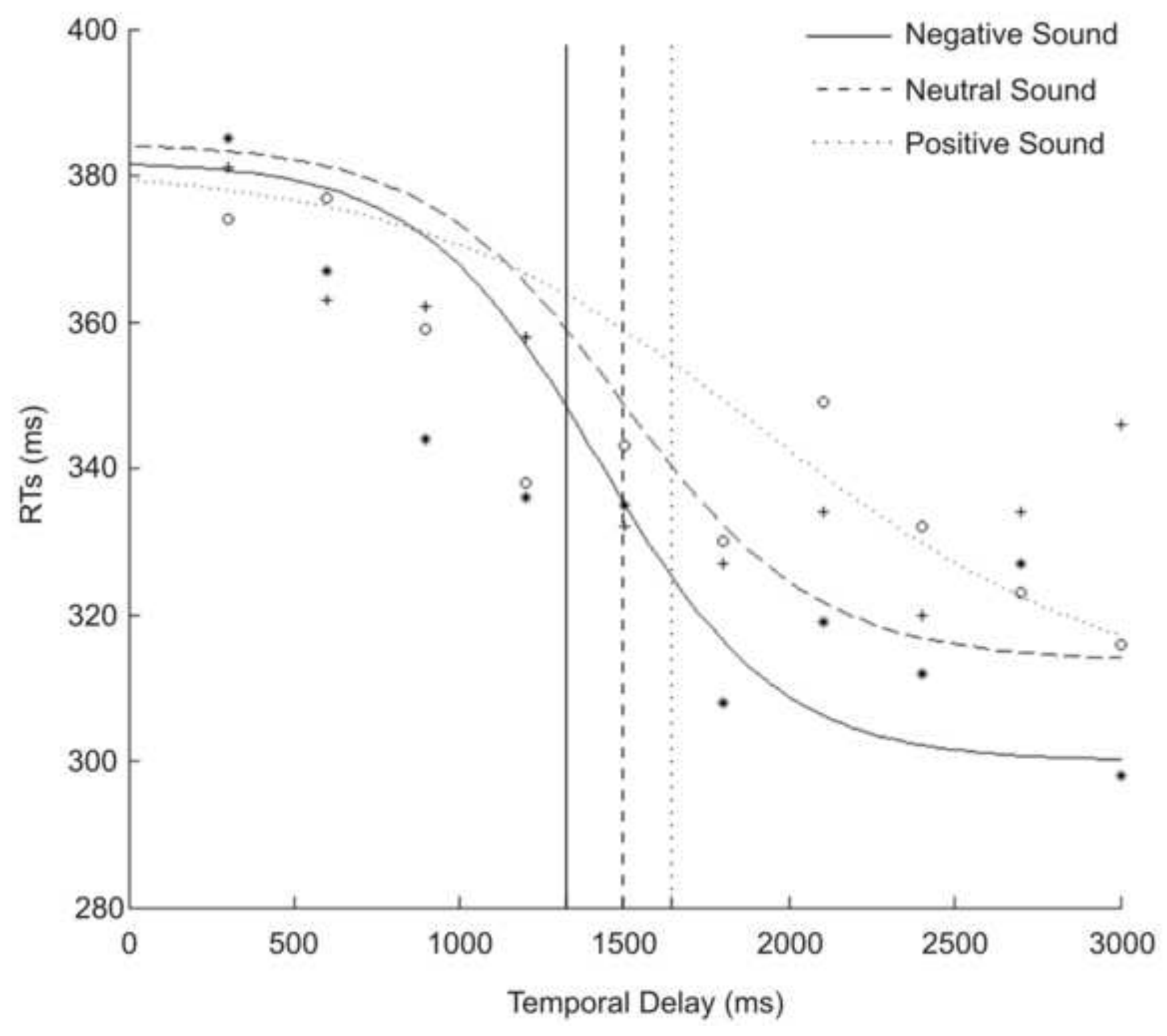

